\title{
Apixaban-Calibrated Anti-FXa Activity in Relation to Outcome Events and Clinical Characteristics in Patients with Atrial Fibrillation: Results from the AVERROES Trial
}

\author{
Vinai C. Bhagirath ${ }^{1,2}$ John W. Eikelboom ${ }^{1,2}$ Jack Hirsh ${ }^{1,2} \quad$ Michiel Coppens $^{2,3}$ Jeffrey Ginsberg ${ }^{1}$ \\ Thomas Vanassche $e^{2,4}$ Fei Yuan ${ }^{2}$ Noel Chan ${ }^{1,2}$ Salim Yusuf ${ }^{1,2}$ Stuart J. Connolly,
}

${ }^{1}$ Department of Medicine, McMaster University, Hamilton, Ontario, Canada

2 Population Health Research Institute, McMaster University, Hamilton, Ontario, Canada

${ }^{3}$ Department of Vascular Medicine, Academic Medical Centre, Amsterdam, The Netherlands

${ }^{4}$ Department of Cardiovascular Sciences, University Hospital, Leuven, Belgium

\author{
Address for correspondence Vinai C. Bhagirath, MD, MSC, \\ Department of Medicine, McMaster University, Hamilton General \\ Hospital, 6th Floor, M-Section, 237 Barton Street E., Hamilton, ON, \\ Canada L9A 1A3 (e-mail: bhagiv@mcmaster.ca).
}

TH Open 2017;1:e139-e145.

\begin{abstract}
Keywords

- apixaban

- fibrillation

- hemorrhage

- systemic embolism

Background In patients with nonvalvular atrial fibrillation (AF), apixaban is given in doses of 5 or $2.5 \mathrm{mg}$ twice daily, according to clinical characteristics. The usual ontreatment range of apixaban drug levels, as determined by apixaban-calibrated antifactor Xa (anti-Xa) activity, has previously been measured in small cohorts; however, the association between anti-Xa activity and clinical outcomes and the predictors of variability in anti-Xa activity have not been well studied in the AF population.

Methods and Results Anti-Xa activity was measured before taking the morning dose, 3 months after enrollment in the AVERROES study using a calibrated anti-Xa assay (Rotachrom). Patients with two of the following criteria-age $>80$; weight $<60 \mathrm{~kg}$; or creatinine $>133 \mu \mathrm{g} / \mathrm{L}-$ received $2.5 \mathrm{mg}$ twice daily $(n=145)$, while all others received $5 \mathrm{mg}$ twice daily $(n=2,247)$. A total of 2,392 patients were included, with median follow-up of 1.1 years. Median apixaban anti-Xa activity was $122 \mathrm{ng} / \mathrm{mL}$ (interquartile range [IQR]: $63-198 \mathrm{ng} / \mathrm{mL}$ ) for the entire group; $99 \mathrm{ng} / \mathrm{mL}$ (IQR: $60-146 \mathrm{ng} / \mathrm{mL}$ ) for the $2.5-\mathrm{mg}$ group; and $125 \mathrm{ng} / \mathrm{mL}$ (IQR: $64-202 \mathrm{ng} / \mathrm{mL}$ ) for the $5-\mathrm{mg}$ group $(p=0.003)$. A relationship was evident between bleeding and anti-Xa activity $(p=0.01)$, which was driven by minor bleeding. No relationship was evident between major bleeding or stroke/systemic embolism and anti-Xa activity. In those receiving the 5-mg dose, estimated glomerular filtration rate, sex, and age had the strongest association with anti-Xa activity.

Conclusion There is considerable variability in anti-Xa activity among AF patients receiving apixaban. Rates of major bleeding and stroke/systemic embolism were low irrespective of anti-Xa activity.

Clinical Trial Registration ClinicalTrials.gov NCT00496769; https://clinicaltrials.gov/ ct2/show/NCT00496769.
\end{abstract}

received

October 13, 2017

accepted after revision

November 6, 2017
DOI https://doi.org/

10.1055/s-0037-1613679. ISSN 2512-9465.
(C) 2017 Georg Thieme Verlag KG Stuttgart - New York
License terms

(c) (1) 


\section{Introduction}

Apixaban, a direct factor Xa (FXa) inhibitor, has been evaluated in two large phase III randomized controlled trials of stroke prevention in patients with atrial fibrillation (AF). ${ }^{1,2}$ The ARISTOTLE trial demonstrated that fixed-dose unmonitored apixaban compared with dose-adjusted warfarin was associated with a similar rate of ischemic stroke, a halving of hemorrhagic stroke, and a one-third reduction in major bleeding. ${ }^{1}$ When compared with aspirin in the AVERROES trial, fixed-dose apixaban reduced the risk of ischemic stroke by one-half, with similar rates of major bleeding and hemorrhagic stroke. $^{2}$ The effects of apixaban relative to warfarin or aspirin were consistent across all subgroups examined, including participants with advanced age, moderate renal impairment, or prior stroke. ${ }^{3-6}$

In both trials, apixaban was administered as $5 \mathrm{mg}$ twice daily unless patients met criteria (two or more of age $\geq 80$ years, weight $<60 \mathrm{~kg}$, and serum creatinine $>1.5 \mathrm{mg} / \mathrm{dL}$ ) for reduced dose apixaban $2.5 \mathrm{mg}$ twice daily. This dosing strategy was associated with low rates of stroke (0.97 and $1.4 \% / y e a r)$ and bleeding (1.4 and 2.13\%/year) in the ARISTOTLE and AVERROES trials, respectively, suggesting that any possible incremental benefit of dose adjustment based on the results of routine drug level monitoring would be marginal. On the other hand, measurement of apixaban levels may be useful in selected situations. These include the management of patients who present with bleeding or acute stroke and in those with clinical characteristics that are associated with a risk of extreme drug levels. In such situations, knowledge of expected drug levels, the relationship between drug levels and clinical events, and determinants of drug levels can help guide clinical decision making.

In the AVERROES pharmacokinetic substudy, apixabancalibrated anti-Xa activity (hereafter referred to as anti-Xa activity) was measured at steady state immediately prior to the morning dose of study drug. Our objectives were (1) to establish the range of usual on-treatment trough apixaban anti-Xa activity; (2) to examine the relationship between trough anti-Xa activity and bleeding and thromboembolic events; and (3) to explore the contribution of various clinical characteristics to apixaban anti-Xa activity.

\section{Materials and Methods}

\section{Trial Design, Outcome Definitions, and Blood Sample Collection}

The AVERROES trial ${ }^{2}$ was designed to determine whether apixaban is superior to aspirin for stroke prevention in patients with $\mathrm{AF}$ and an additional risk factor for stroke $\left(\mathrm{CHADS}_{2}\right.$ score $\geq 1$ or with documented peripheral arterial disease) $)^{7}$ who were unsuitable for vitamin K antagonist therapy. The trial included 5,599 patients, of whom 2,808 received apixaban in a blinded, double-dummy design. Patients who met two of the three following criteria-age $\geq 80$ years, body weight $\leq 60 \mathrm{~kg}$, or serum creatinine $\geq 133 \mu \mathrm{mol} / \mathrm{L}(1.5 \mathrm{mg} / \mathrm{dL}$ )-received a study dose of $2.5 \mathrm{mg}$ apixaban twice daily, while all others received $5 \mathrm{mg}$ twice daily. To distinguish thromboembolic events from bleeding events in our analyses, stroke included ischemic and unspecified stroke but excluded hemorrhagic stroke, and vascular death excluded fatal bleeding. Bleeding was defined according to the definition of International Society on Thrombosis and Haemostasis (ISTH). ${ }^{8}$ All outcome events were adjudicated by a committee blinded to the treatment arm. Compliance was estimated using pill counts which were performed at each study visit. Estimated glomerular filtration rate (eGFR) was calculated using the CKD-EPI formula. ${ }^{9}$ The trial was stopped early after a median follow-up of 1.1 years due to clear evidence of superiority of apixaban.

Follow-up visits were scheduled at 1 and 3 months after randomization and every 3 months thereafter. Blood samples for this study were collected at the 3-month visit. Patients were asked to hold the dose of study drug preceding the visit so that the blood sample was taken prior to the morning dose. Citrated blood was double spun to produce platelet poor plasma, aliquoted, and centrally stored at $-80^{\circ} \mathrm{C}$ for future analyses. Apixaban has previously been shown to remain stable at $-70^{\circ} \mathrm{C}$ for up to 2 years, ${ }^{10}$ and all samples were tested within this time frame.

The AVERROES trial was approved by all appropriate regulatory authorities and ethics committees (ClinicalTrials.gov NCT004969769). All patients provided written informed consent before study entry.

\section{Measurement of Plasma Apixaban-Calibrated Anti-FXa Activity}

Anti-Xa activity was measured by a chromogenic anti-factor Xa assay (Rotachrom Heparin, Diagnostica Stago S.A.S, Asnièressur-Seine, France). Since apixaban calibrators were not commercially available at the time the assays were done, apixaban calibrators were prepared by crushing apixaban tablets and dissolving in DMSO, as per Barrett et al. ${ }^{10}$ Results were calibrated against a standard curve developed from these calibrators $(500-15.6 \mathrm{ng} / \mathrm{mL})$. The standard curve remained linear down to $15.6 \mathrm{ng} / \mathrm{mL}$, which was therefore considered as the lower limit of quantitation of the assay. Values below this limit were transposed to $15.6 \mathrm{ng} / \mathrm{mL}$ for the purpose of statistical analysis. All calibrators and patient samples were measured on a STA-Compact instrument (Diagnostica Stago S.A.S.). The antiXa assays were performed by Hemostasis Reference Laboratories (Hamilton, Ontario, Canada).

\section{Statistical Analysis}

Incidence of clinical outcomes is expressed per 100 patientyears. Comparisons between apixaban dose groups (5-mg twice daily and 2.5-twice daily) were performed using Wilcoxon's rank-sum test for continuous variables and by chi-squared test for categorical variables. Associations between outcomes and anti-Xa activity were determined using a Cox proportional hazards model. For the graphical display of these associations, logistic regression was used to estimate the probability of an event for each anti-Xa activity value. Analyses of the association between anti-Xa activity and clinical variables were restricted to patients receiving the 5-mg twice-daily dose. Correlations between anti-Xa activity and continuous variables were calculated by Spearman's correlation, while associations between 
anti-Xa activity and binary variables were examined by Wilcoxon's rank-sum test. Independent predictors of anti-Xa levels were determined by stepwise multiple linear regression with backward elimination. Only patients with available compliance data were included in the compliance analyses. Compliance rates for those in the lowest anti-Xa activity decile were compared against those in the upper nine deciles by Wilcoxon's rank-sum test. All analyses were performed using SAS version 9.4 software (SAS Institute, Clary, North Carolina, United States).

\section{Results}

\section{Clinical Characteristics of Study Population}

Blood samples were available for 2,392 (85\%) of 2,808 participants randomized to apixaban, of whom 2,247 received the 5-mg twice-daily dose and 145 received the 2.5 -mg twice-daily dose. Clinical characteristics of tested patients are presented in -Table 1. Patients receiving the $2.5-\mathrm{mg}$ twice-daily dose were older, had lower body mass, had lower eGFR, were more likely to be female, had higher $\mathrm{CHADS}_{2}$ score, and were less likely to be taking a moderate or strong inhibitor of P-glycoprotein (Pgp) or CYP3A4 than those receiving the 5-mg twice-daily dose $(p<0.05$ for each comparison; - Table 1).

\section{Apixaban-Calibrated Anti-FXa Activity}

The median anti-Xa activity was $122 \mathrm{ng} / \mathrm{mL}$ (interquartile range [IQR]: 63-198 ng/mL) and the interindividual geometric coefficient of variation (GCV) was $113 \%$. In 230 (9.7\%) of the patients, the anti-Xa activity was below the lower limit of quantitation of the calibrated anti-factor Xa assay $(15.6 \mathrm{ng} / \mathrm{mL})$. The 10 th to 90 th centile range was 17 to $289 \mathrm{ng} / \mathrm{mL}$. Patients receiving the $2.5-\mathrm{mg}$ twice-daily dose had a $21 \%$ lower median anti-Xa activity compared with those receiving the 5-mg twice-daily dose

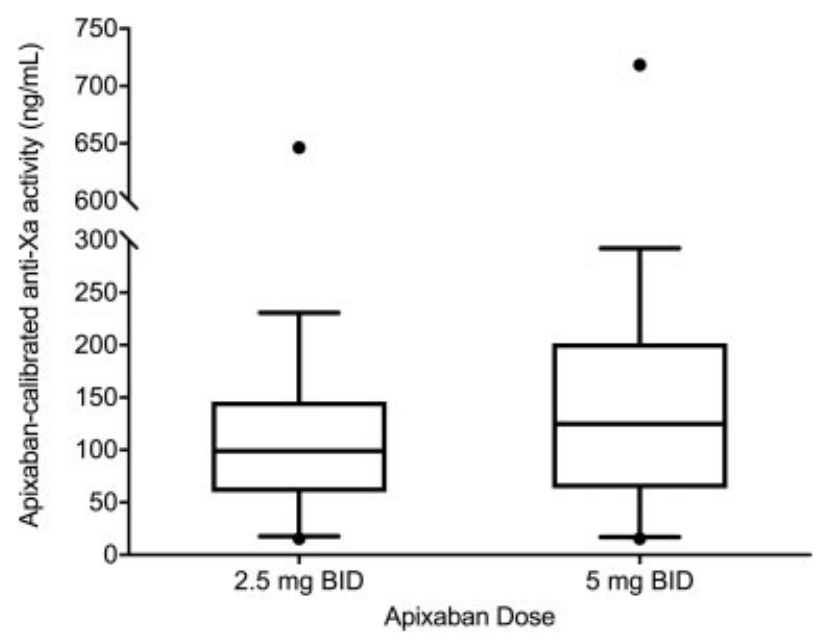

Fig. 1 Apixaban-calibrated anti-Xa activity by dose. Blood samples were drawn immediately prior to the morning dose of apixaban. Boxes represent median and interquartile range, whiskers represent 10th to 90 th centiles, and points represent minimum and maximum.

(99 ng/mL, IQR: 60-146, 10th to 90th centile range: 18-231 vs. $125 \mathrm{ng} / \mathrm{mL}$, IQR: 64-202, 10th to 90th centile range: 17-292; $p=0.003$ for comparison of medians; - Fig. 1).

\section{Anti-Xa Activity and Clinical Outcomes}

The rates of clinical outcomes according to quintile of anti-Xa activity are presented in -Table $\mathbf{2}$. No significant association was found between anti-Xa activity and stroke/systemic embolism ( Fig. 2A), myocardial infarction, or symptomatic pulmonary embolism ( $p \geq 0.44$ for each), although event rates were low, limiting power to detect a relationship. In a post hoc analysis, patients within the lowest decile of anti-Xa

Table 1 Apixaban anti-Xa activity $(\mathrm{ng} / \mathrm{mL})$ and clinical characteristics for the entire group and according to apixaban dose

\begin{tabular}{|l|l|l|l|l|}
\hline Apixaban dose & $\begin{array}{l}\text { Either dose } \\
(\boldsymbol{n}=2,392)\end{array}$ & $\begin{array}{l}2.5 \mathrm{mg} \text { BID } \\
(\boldsymbol{n}=145)\end{array}$ & $\begin{array}{l}\mathbf{5} \mathrm{mg} \text { BID } \\
(\boldsymbol{n}=\mathbf{2 , 2 4 7})\end{array}$ & $\mathbf{p}$-Value \\
\hline Median anti-Xa activity $(\mathrm{ng} / \mathrm{mL})$ & $122(63-198)$ & $99(60-146)$ & $125(64-202)$ & 0.003 \\
\hline Age $(\mathrm{y})$ & $70(63-76)$ & $84(81-86)$ & $69(62-75)$ & $<0.001$ \\
\hline Weight $(\mathrm{kg})$ & $78(66-91)$ & $56(50-60)$ & $79(68-92)$ & $<0.001$ \\
\hline eGFR $\left(\mathrm{mL} / \mathrm{min} / 1.73 \mathrm{~m}^{2}\right)$ & $71(54-92)$ & $38(32-46)$ & $73(57-94)$ & $<0.001$ \\
\hline Female sex & $969(41 \%)$ & $95(66 \%)$ & $874(39 \%)$ & $<0.001$ \\
\hline History of heart failure & $954(40 \%)$ & $56(39 \%)$ & $898(40 \%)$ & 0.75 \\
\hline CHADS score 0-1 & $869(36.3 \%)$ & $14(10 \%)$ & $855(38 \%)$ & $<0.001$ \\
\hline CHADS score 2 & $896(37.5 \%)$ & $62(43 \%)$ & $834(37 \%)$ & $557(25 \%)$ \\
\hline CHADS score $>2$ & $626(26.2 \%)$ & $69(48 \%)$ & $530(22.6 \%)$ & 0.032 \\
\hline $\begin{array}{l}\text { Concomitant use of CYP3A4 or } \\
\text { Pgp inhibitor (moderate or strong) }\end{array}$ & $553(23.2 \%)$ & $23(15.9 \%)$ & $137(6.1 \%)$ & 0.78 \\
\hline $\begin{array}{l}\text { Liver disease (baseline elevation } \\
\text { of bilirubin, AST, or ALT) }\end{array}$ & $145(6.1 \%)$ & $8(5.5 \%)$ & & \\
\hline
\end{tabular}

Abbreviations: ALT, alanine aminotransferase; AST, aspartate aminotransferase; CYP3A4, cytochrome P450 3A4; eGFR, estimated glomerular filtration rate; Pgp, P-glycoprotein.

Notes: Values presented as median (interquartile range) or number (\%). p-Value is for comparison between doses by Wilcoxon's rank-sum for continuous variables and chi-squared test for categorical variables. Analysis by concomitant use of a CYP3A4 or Pgp inducer (moderate or strong) is not included due to small numbers $(n=5)$. 
e142 Apixaban Level in Relation to Clinical Outcomes Bhagirath et al.

Table 2 Clinical events by apixaban anti-Xa activity quintile

\begin{tabular}{|c|c|c|c|c|c|c|}
\hline & Quintile 1 & Quintile 2 & Quintile 3 & Quintile 4 & Quintile 5 & $\begin{array}{l}\text { p-Value } \\
\text { (Cox-regression) }\end{array}$ \\
\hline Range of anti-Xa activity: $\mathrm{ng} / \mathrm{mL}$ & $0-50$ & $51-98$ & $99-146$ & $147-254$ & $255-719$ & \\
\hline \multicolumn{7}{|c|}{ Embolic or thrombotic events: $n$ (/100 patient-years) } \\
\hline $\begin{array}{l}\text { Ischemic/unspecified } \\
\text { stroke or systemic embolism }\end{array}$ & $10(1.82)$ & $2(0.37)$ & $2(0.38)$ & $6(1.08)$ & $4(0.76)$ & 0.44 \\
\hline $\mathrm{PE}$ & $0(0)$ & $2(0.37)$ & $2(0.38)$ & $0(0)$ & $0(0)$ & 0.55 \\
\hline MI & $5(0.91)$ & $2(0.37)$ & $3(0.57)$ & $6(1.08)$ & $1(0.19)$ & 0.50 \\
\hline \multicolumn{7}{|c|}{ Bleeding events: $n$ (/100 patient-years) } \\
\hline Any bleeding & $35(6.68)$ & $50(9.82)$ & $51(10.33)$ & $61(11.84)$ & $59(11.94)$ & 0.01 \\
\hline Major bleeding & $6(1.09)$ & $5(0.91)$ & $11(2.12)$ & $5(0.90)$ & $7(1.33)$ & 0.58 \\
\hline $\begin{array}{l}\text { Clinically relevant } \\
\text { nonmajor bleeding }\end{array}$ & $12(2.20)$ & $15(2.80)$ & $12(2.33)$ & $20(3.67)$ & $14(2.68)$ & 0.62 \\
\hline Minor bleeding & $19(3.55)$ & $36(6.92)$ & $32(6.34)$ & $39(7.39)$ & $41(8.19)$ & $<0.01$ \\
\hline Gl bleeding & $3(0.54)$ & $1(0.18)$ & $3(0.58)$ & $0(0)$ & $3(0.57)$ & 1.00 \\
\hline \multicolumn{7}{|l|}{ Death: $n$ (/100 patient-years) } \\
\hline Total death & $14(2.53)$ & $14(2.55)$ & $14(2.67)$ & $14(2.50)$ & $16(3.02)$ & 0.23 \\
\hline Vascular death & $9(1.62)$ & $9(1.64)$ & $12(2.29)$ & $11(1.97)$ & $14(2.64)$ & 0.05 \\
\hline Fatal bleeding & 0 & 0 & $2(0.382)$ & 0 & $1(0.189)$ & 0.50 \\
\hline
\end{tabular}

Abbreviations: Gl, gastrointestinal; MI, myocardial infarction; PE, pulmonary embolism.

Notes: Values for outcomes represent total number of events (events/hundred patient-years). $p$-Value is for correlation between outcome and apixaban anti-Xa activity in a Cox regression model.

activity had a significantly greater risk of stroke than those with higher anti-Xa activity (Fisher's exact test, $p=0.013$ ). There was an association between anti-Xa activity and occurrence of bleeding (of any severity; $p=0.01$ by Cox regression; - Fig. 2B). This was driven mainly by minor bleeding ( $p=0.009$ by Cox regression), with rates of 3.55 , $6.92,6.34,7.39$, and 8.19 per 100 patient-years for the lowest to highest quintiles, respectively, of anti-Xa activity. The rates of major bleeding were low, and there was no detected association between anti-Xa activity and major bleeding in a Cox regression analysis $(p=0.581)$. Similarly, no association was detected with clinically relevant nonmajor bleeding ( $p=0.621$ by Cox regression; - Table 2 ).

\section{Effect of Clinical Characteristics on Anti-Xa Activity}

We evaluated the clinical predictors of trough apixaban anti-Xa activity in the 2,247 patients who received the 5-mg twice-daily dose. Increasing age correlated with increased anti-Xa activity $(p<0.001)$. Female sex, history of diabetes, and use of a CYP3A4 or Pgp inhibitor (moderate or strong) were also associated with higher anti-Xa activity $(p<0.001, p=0.011$, and $p=0.002$, respectively). Body weight and eGFR were negatively correlated with anti-Xa activity ( $p=0.002$ and $p<0.001$, respectively), and history of heart failure was associated with lower anti-Xa activity ( $p<0.001 ;-$ Table 3 ). In the stepwise multiple linear regression model, eGFR, female sex, history of heart failure, body weight, age, and use of moderate or strong inhibitors of CYP3A4 or Pgp remained independent significant determinants of anti-Xa activity $(p<0.05)$.

\section{Compliance Analyses}

A compliance analysis was performed in 2,062 patients (86\%) with available compliance data. Compliance was lower in patients in the lowest decile of anti-Xa activity compared with the upper nine deciles (94.6\%, IQR: 80.4-98.6\% vs. 96.5\%, IQR: 90.3-99.1\%; $p<0.001$ by Wilcoxon's rank-sum test). When including only those patients with $\geq 80 \%$ compliance $(n=1822)$, anti-Xa activity was no longer positively correlated to risk of minor bleeding by Cox regression $(p=0.212)$. The difference in risk of stroke between patients within the lowest decile of anti-Xa activity and those with higher anti-Xa activity was no longer statistically significant (Fisher's exact test, $p=0.070$ ).

\section{Discussion}

We performed this substudy of AVERROES to examine three items: (1) the range of on-treatment trough levels; (2) the relationship between trough levels and clinical events; and (3) clinical predictors influencing trough levels in AF patients taking apixaban. Our findings either extend or provide new information on all three.

\section{Range of On-Treatment Levels}

Previous data on the range of usual on-treatment levels in AF patients treated with apixaban were limited to predicted range from pharmacokinetic-pharmacodynamic modeling or to small studies. ${ }^{11-13}$ Our study provides more accurate and precise estimates than those obtained from predicted 

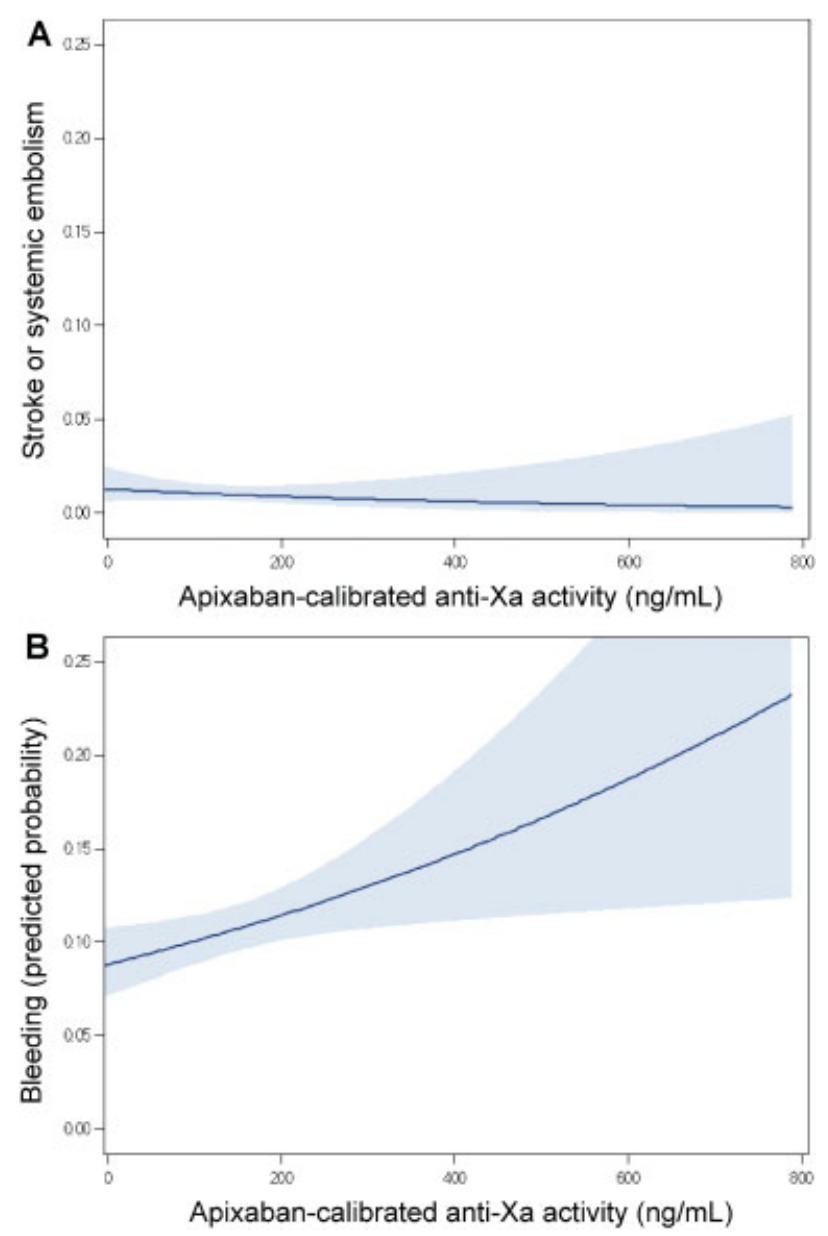

Fig. 2 Stroke/systemic embolism and bleeding versus anti-Xa activity. Predicted probability of (A) stroke/systemic embolism; or (B) bleeding of any severity (minor, clinically relevant nonmajor, or major) versus anti-Xa activity. The shaded region represents the $95 \%$ confidence interval.

models or from small studies, respectively, because samples were taken from a much larger population of AF patients $(n=2,392)$ taking apixaban. The range of levels found in our study is in keeping with the interpatient variability in drug levels observed with other direct oral anticoagulants. ${ }^{14,15}$ Patients receiving the reduced dose of $2.5-\mathrm{mg}$ twice-daily had approximately 20\% lower median and 90th centile of anti-Xa activity as those receiving 5-mg twice-daily, but the lowest decile of both groups was very similar (-Fig. 1). Since the clinical criteria for reduced dose apixaban were chosen because they had been shown to be associated with increased drug activity as well as an increased risk of bleeding, ${ }^{16}$ the current dosing recommendations seem to have appropriately resulted in decreasing the proportion of patients with high drug levels in this group, without increasing the proportion with the lowest levels. Our observations support the use of the currently employed clinical criteria for dose reduction. A previously published registry reported that a significant proportion of patients taking apixaban for AF were prescribed the 2.5 -mg twice-daily dose when they did not meet criteria for dose reduction as specified in AVERROES and ARISTOTLE. ${ }^{17}$ Our data do not provide direct information on the drug levels or risk of clinical events in such patients.

Relationship between Trough Level and Clinical Events Based on the RE-LY (dabigatran) and ENGAGE AF-TIMI 48 (ENGAGE-AF, edoxaban) trials, ${ }^{14,15}$ we expected to find relationships between apixaban trough anti-Xa activity and the risk of both major bleeding and of stroke/SEE. In both the RE-LYand ENGAGE-AF subanalyses, the risk of major bleeding increased with trough level, but an increased risk of stroke/SEE was observed only when levels fell into low extremes. We observed a significant relationship between trough levels and total bleeding, but not with major or clinically relevant bleeding. This may be explained by the fact that both RE-LY and ENGAGE-AF had considerably more events ( $>10$ times the number of embolic and bleeding events in ENGAGE-AF compared with AVERROES), and therefore power to detect relationships. The lack of strict timing of blood sampling in our study, the inclusion of events before and after blood sampling, and the use of anti-Xa activity rather than liquid chromatography with tandem mass spectrometry may also have reduced our ability to detect such relationships. However, our finding that the relationship between stroke/SEE and levels was observed only when trough apixaban anti-Xa activity fell below the lowest decile is consistent with the findings reported in RE-LY and ENGAGE-AF. About $90 \%$ of patients in the lowest decile had anti-Xa activity below the level of detection, of whom $24 \%$ had compliance less than $80 \%$. We cannot rule out the possibility that the relationships seen for minor bleeding and stroke with anti-Xa activity were driven by patients with absent drug levels due to noncompliance.

\section{Clinical Predictors of Trough Levels}

In an analysis restricted to patients taking $5 \mathrm{mg}$ BID, we found renal function, age, sex, body weight, diabetes, heart failure, and use of inhibitors of CYP3A4 or P-gp to be independent clinical predictors of trough apixaban levels. Of these seven clinical factors, renal function had the most important influence on apixaban levels, followed by age, sex, and weight. As shown in - Table 3, as eGFR varied from $\geq 80$ to $\leq 30 \mathrm{~mL} / \mathrm{min}$, median trough apixaban level increased more than 2.5 -fold from 105 to $274 \mathrm{ng} / \mathrm{mL}$. This finding was unexpected because in selecting patients taking $5 \mathrm{mg}$ BID, we excluded those who had extreme values for three recognized criteria (age $\geq 80$ years, weight $<60 \mathrm{~kg}$, and serum creatinine $>1.5 \mathrm{mg} / \mathrm{dL}$ ) of an increased response to apixaban. Such a finding could be important clinically because it raises the possibility that the currently accepted clinical criteria for dose reduction could be improved by including either eGFR $\leq 30 \mathrm{~mL} / \mathrm{min}$ as an independent criterion for dose reduction or by developing a weighted score system based on the identified clinical predictors (renal function, age, sex, body weight). The small number of patients taking the 2.5 -mg twice-daily dose limited our ability to separately analyze this subgroup.

Our findings indicate that apixaban is effective and safe across a broad range of anti-Xa levels and provides no support for routine laboratory monitoring. Knowledge of 
Table 3 Effect of clinical characteristics on apixaban anti-Xa activity

\begin{tabular}{|l|l|l|l|l|l|}
\hline Characteristic & Subgroup 1 & Subgroup 2 & Subgroup 3 & Subgroup 4 & $p$-Value \\
\hline Age $(\mathrm{y})$ & $<65(n=737)$ & 65 to $<80(n=1,268)$ & $\geq 80(n=242)$ & - & \\
\hline Anti-Xa activity & $101(47-168)$ & $131(71-208)$ & $166(107-255)$ & - & $<0.001$ \\
\hline Weight $(\mathrm{kg})$ & $>100(n=321)$ & $>60$ to $100(n=1,668)$ & $\leq 60(n=226)$ & - & \\
\hline Anti-Xa activity & $115(65-167)$ & $126(65-204)$ & $135(46-274)$ & - & 0.002 \\
\hline Sex & Male $(n=1,373)$ & Female $(n=874)$ & - & - & \\
\hline Anti-Xa activity & $115(61-183)$ & $141(70-232)$ & - & - & $<0.001$ \\
\hline CHADS 2 & $0-1(n=855)$ & $2(n=834)$ & $>2(n=557)$ & - & \\
\hline Anti-Xa activity & $121(68-188)$ & $119(57-204)$ & $136(64-224)$ & - & 0.066 \\
\hline Heart failure & Yes $(n=898)$ & No $(n=1,348)$ & - & - & \\
\hline Anti-Xa activity & $105(48-194)$ & $133(75-207)$ & - & - & $<0.001$ \\
\hline eGFR (mL/min/1.73 $\left.\mathrm{m}^{2}\right)$ & $\geq 80(n=931)$ & 50 to $<80(n=962)$ & 30 to $<50(n=317)$ & $<30(n=17)$ & \\
\hline Anti-Xa activity & $105(53-163)$ & $133(71-212)$ & $177(86-294)$ & $274(136-424)$ & $<0.001$ \\
\hline Diabetes & Yes $(n=433)$ & No $(n=1,813)$ & - & - & \\
\hline Anti-Xa activity & $134(79-208)$ & $122(61-199)$ & - & - & 0.011 \\
\hline $\begin{array}{l}\text { Concomitant use of } \\
\text { (YP3A4 or Pgp inhibitor } \\
\text { (moderate or strong) }\end{array}$ & Yes $(n=530)$ & No $(n=1,717)$ & - & - & \\
\hline Anti-Xa activity & $141(68-225)$ & $119(63-194)$ & - & & \\
\hline $\begin{array}{l}\text { Liver disease } \\
\text { baseline elevation of } \\
\text { bilirubin, AST, or ALT) }\end{array}$ & Yes $(n=137)$ & No $(n=2,110)$ & - & - & \\
\hline Anti-Xa activity & $118(56-185)$ & $125(64-203)$ & - & - & \\
\hline
\end{tabular}

Abbreviations: ALT, alanine aminotransferase; AST, aspartate aminotransferase; eGFR, estimated glomerular filtration rate.

Notes: Analysis is for patients receiving 5-mg twice-daily dose only. Anti-Xa activity is median apixaban-calibrated anti-FXa activity in $\mathrm{ng} / \mathrm{mL}$ (interquartile range). $p$-Value is for Spearman's correlation for continuous variables and Wilcoxon's rank-sum test for binary variables. Analysis by concomitant use of a CYP3A4 or Pgp inducer (moderate or strong) is not included due to small numbers $(n=5)$.

usual on-treatment drug levels should also be helpful when managing patients taking one or more drugs that interact with apixaban; high or low apixaban levels in this situation could prompt clinicians to change drug therapy to ensure that patients remain effectively and safely protected against the risk of stroke.

\section{Funding}

The AVERROES study was sponsored by Bristol-Myers Squibb and Pfizer.

\section{Conflict of Interest}

None declared.

\section{References}

1 Granger CB, Alexander JH, McMurray JJ, et al; ARISTOTLE Committees and Investigators. Apixaban versus warfarin in patients with atrial fibrillation. N Engl J Med 2011;365(11):981-992

2 Connolly SJ, Eikelboom J, Joyner C, et al; AVERROES Steering Committee and Investigators. Apixaban in patients with atrial fibrillation. N Engl J Med 2011;364(09):806-817

3 Halvorsen S, Atar D, Yang H, et al. Efficacy and safety of apixaban compared with warfarin according to age for stroke prevention in atrial fibrillation: observations from the ARISTOTLE trial. Eur Heart J 2014;35(28):1864-1872

$4 \mathrm{Ng} \mathrm{KH}$, Shestakovska O, Connolly SJ, et al. Efficacy and safety of apixaban compared with aspirin in the elderly: a subgroup analysis from the AVERROES trial. Age Ageing 2016;45(01):7783

5 Hohnloser SH, Hijazi Z, Thomas L, et al. Efficacy of apixaban when compared with warfarin in relation to renal function in patients with atrial fibrillation: insights from the ARISTOTLE trial. Eur Heart J 2012;33(22):2821-2830

6 Easton JD, Lopes RD, Bahit MC, et al; ARISTOTLE Committees and Investigators. Apixaban compared with warfarin in patients with atrial fibrillation and previous stroke or transient ischaemic attack: a subgroup analysis of the ARISTOTLE trial. Lancet Neurol 2012;11(06):503-511

7 Gage BF, Waterman AD, Shannon W, Boechler M, Rich MW, Radford MJ. Validation of clinical classification schemes for predicting stroke: results from the National Registry of Atrial Fibrillation. JAMA 2001;285(22):2864-2870

8 Schulman S, Kearon C; Subcommittee on Control of Anticoagulation of the Scientific and Standardization Committee of the International Society on Thrombosis and Haemostasis. Definition of major bleeding in clinical investigations of antihemostatic medicinal products in non-surgical patients. J Thromb Haemost 2005;3(04):692-694

9 Levey AS, Stevens LA, Schmid CH, et al; CKD-EPI (Chronic Kidney Disease Epidemiology Collaboration). A new equation to estimate glomerular filtration rate. Ann Intern Med 2009;150(09):604-612 
10 Barrett YC, Wang Z, Frost C, Shenker A. Clinical laboratory measurement of direct factor Xa inhibitors: anti-Xa assay is preferable to prothrombin time assay. Thromb Haemost 2010; 104(06):1263-1271

11 Kowalsk K, Nielsen J, Roy A, et al. Apixaban exposure and anti-Xa activity in nonvalvular atrial fibrillation patients: an application of population PK/PD analysis. J Pharmacokinet Pharmacodyn 2014;41:S7-S101. Abstract M-027

12 Osanai H, Ajioka M, Masutomi T, et al. Measurement of anti-factor Xa activity in patients on apixaban for non-valvular atrial fibrillation. Circ J 2015;79(12):2584-2590

13 Testa S, Tripodi A, Legnani C, et al; START-Laboratory Register. Plasma levels of direct oral anticoagulants in real life patients with atrial fibrillation: results observed in four anticoagulation clinics. Thromb Res 2016;137:178-183

14 Reilly PA, Lehr T, Haertter S, et al; RE-LY Investigators. The effect of dabigatran plasma concentrations and patient characteristics on the frequency of ischemic stroke and major bleeding in atrial fibrillation patients: the RE-LY Trial (Randomized Evaluation of Long-Term Anticoagulation Therapy). J Am Coll Cardiol 2014;63 (04):321-328

15 Ruff CT, Giugliano RP, Braunwald E, et al. Association between edoxaban dose, concentration, anti-Factor Xa activity, and outcomes: an analysis of data from the randomised, double-blind ENGAGE AF-TIMI 48 trial. Lancet 2015;385(9984):22882295

16 Hanna MS, Mohan P, Knabb R, Gupta E, Frost C, Lawrence JH. Development of apixaban: a novel anticoagulant for prevention of stroke in patients with atrial fibrillation. Ann N Y Acad Sci 2014; 1329:93-106

17 Steinberg BA, Shrader P, Thomas L, et al; ORBIT-AF Investigators and Patients. Off-label dosing of non-vitamin $\mathrm{K}$ antagonist oral anticoagulants and adverse outcomes: The ORBIT-AF II Registry. J Am Coll Cardiol 2016;68(24):2597-2604 